\title{
Settling properties of aerobic granular sludge (AGS) and aerobic granular sludge molasses (AGSM)
}

\author{
Azlina Mat Saad ${ }^{1}$, Farrah Aini Dahalan ${ }^{1 *}$, Naimah Ibrahim $^{1}$, Sara Yasina Yusuf ${ }^{1}$, Siti \\ Aqlima $\mathrm{Ahmad}^{2}$, and Khalilah Abdul Khalil ${ }^{3}$ \\ ${ }^{1}$ School of Environmental Engineering, Universiti Malaysia Perlis, Kompleks Pengajian Jejawi 3, \\ 02600 Arau, Perlis, Malaysia \\ ${ }^{2}$ Department of Biochemistry, Faculty of Biotechnology and Biomolecular Sciences, University Putra \\ Malaysia, UPM 43400 Serdang, Selangor, Malaysia \\ ${ }^{3}$ Department of Biomolecular Science, Faculty of Applied Sciences, Universiti Teknologi MARA, \\ Seksyen 2, 40450 Shah Alam, Selangor, Malaysia
}

\begin{abstract}
Aerobic granulation technology is applied to treat domestic and industrial wastewater. The Aerobic granular sludge (AGS) cultivated has strong properties that appears to be denser and compact in physiological structure compared to the conventional activated sludge. It offers rapid settling for solid:liquid separation in wastewater treatment. Aerobic granules were developed using sequencing batch reactor (SBR) with intermittent aerobic - anaerobic mode with 8 cycles in $24 \mathrm{hr}$. This study examined the settling velocity performance of cultivated aerobic granular sludge (AGS) and aerobic granular sludge molasses (AGSM). The elemental composition in both AGS and AGSM were determined using Xray fluorescence (XRF). The results showed that AGSM has higher settling velocity $30.5 \mathrm{~m} / \mathrm{h}$ compared to AGS.
\end{abstract}

\section{Introduction}

Technology on wastewater treatment has improved over time based on various advanced processes to treat municipal and industrial wastewaters. Since the operational performance of the conventional activated sludge processes is limited by the aptitude of a clarifier for the solid;liquid separation of the activated sludge from the treated wastewater, Aerobic granular sludge (AGS) technology is an attractive option due to its settleability advantages. It has become an alternative to the conventional wastewater treatment method specifically activated sludge due to the excellent settling performance and high capacity for biomass retention. With high biomass aggregation of various indigenous microorganisms in the wastewater, AGS exhibits high capability to withstand extensive organic and inorganic loads and demonstrated to have high potential to treat various types of wastewaters [1].

According to [2] aerobic granulation is a combination of two processes involving biological and chemical engineering to form a very compact structure of activated sludge

\footnotetext{
* Corresponding author: farrahaini@unimap.edu.my
} 
that possess high density. Physical dynamism such as vigorous aeration which contributes to effective hydrodynamic shear force has also influenced the acceleration on the formation of AGS. Preferably, a relative high shear force was found to favour rapid granulation.

In general, aerobic granulation involves a highly complex ecosystem which integrates physical, chemical and biological components synergistically to serve for a hybrid compact wastewater treatment system. To achieve optimum process, sequencing batch reactor (SBR) system has been creditably used as an ideal system to develop AGS [3]. During SBR process, activated sludge which is used as seed sludge is subjected to optimized physicochemical growth condition. This is significant to boost vital aggregation of microorganism consortia facultatively to form AGS naturally via cell-to-cell biochemical mechanisms without supplement of any artificial carrier for attached growth [4]. Basically, the development of AGS beginning from the transformation of seed sludge to flocs, and followed by the establishment of sludge granules.

Owing to the constructed design of the aerobic granulation system, the AGS produced have shown perceptible features on the morphology and settling velocity performance surpasses the conventional activated sludge. The structure of the AGS produced are normally possess notable characteristics which appears to be regular, dense, strong, compact, smooth, and uniform in shape and sizes.

However, so far, information about the enhancement of AGS using molasses wastewater and the settling performance is still sparse. Therefore, this study focuses to investigate the settling performance of AGS and AGSM cultivated initially under favourable SBR configuration using synthetic wastewater prior to acclimatization and further granulation in molasses wastewater. In addition, X-ray fluorescence (XRF) analysis was also performed in order to examine the chemical compounds occurred in both AGS and AGSM.

\section{Methodology}

\subsection{Research framework}

AGS was cultivated using SBR in the laboratory. A three-litre cylindrical column reactor was used and operated for 24 hours continuously with intermittent aerobic-anaerobic mode. Fresh activated sludge was collected from local wastewater treatment plant and used as seed sludge to initiate the cultivation process of AGS in SBR.

The cultured AGS were then acclimatized and enriched with molasses wastewater in conical flask under aerobic condition. AGS enriched in molasses wastewater are then known as aerobic granular sludge molasses (AGSM). The characteristics of activated sludge were investigated using APHA Standard Method [5]. The experimental workflow of the development of AGS and AGSM is presented in Fig. 1.

The physical performance and chemical characteristics of the AGS and AGSM were investigated. In this study, settling properties of both AGS and AGSM were significantly studied base on the gravitational settling velocity in order to determine the settleability rate which depict the feasibility of the system in solid:liquid separation performance. The elemental composition of AGS and AGSM were investigated by using XRF spectrometry. 


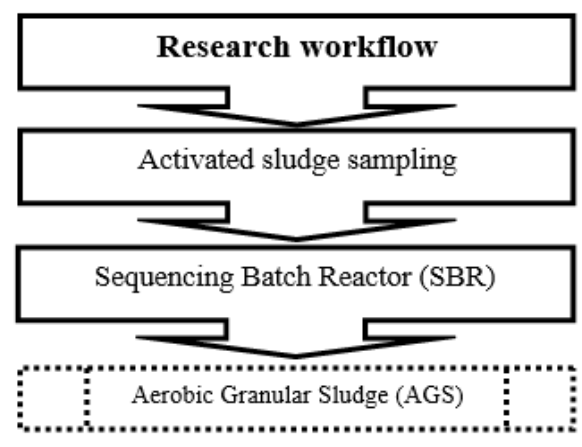

Enrichment of AGS using molasses

Aerobic Granular Sludge Molasses (AGSM)

Fig. 1. Development of AGS and AGSM.

\subsection{Bioreactor set-up and operation}

The SBR used for cultivating aerobic granules was designed for a working volume of 2.4 $\mathrm{L}$, with maximum height of $30.5 \mathrm{~cm}$ and $9 \mathrm{~cm}$ of internal diameter. The volumetric exchange ratio was fixed at $50 \%$. Fig. 2 shows the schematic diagram of the SBR set-up used in this study.

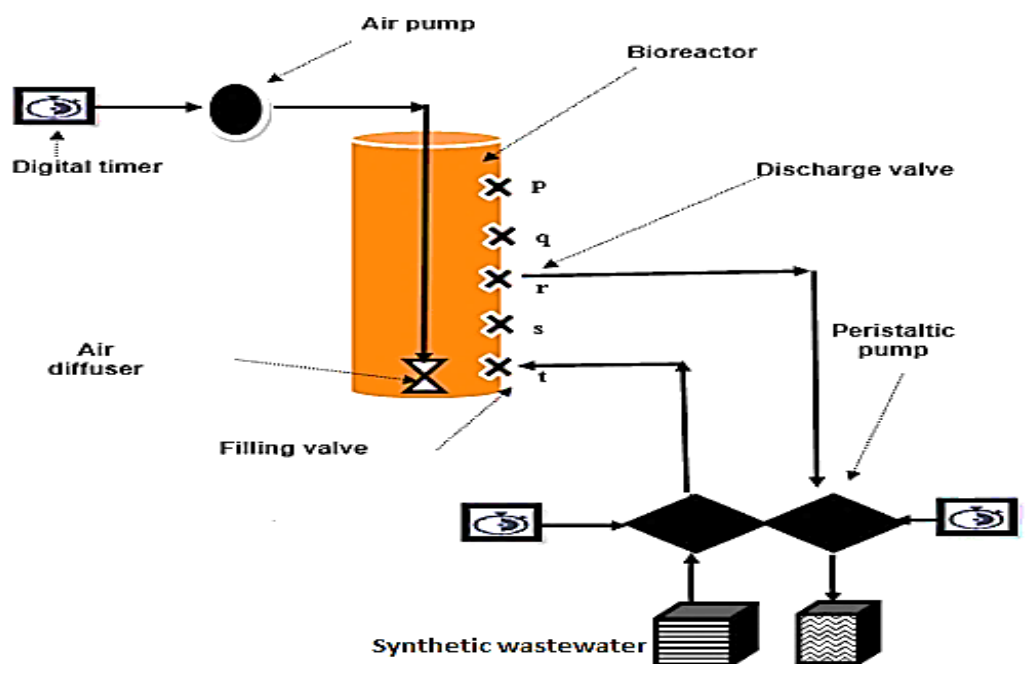

Fig. 2. Bioreactor Set-up.

The bioreactor was operated for 24 hours continuosly in complete cycle of 3 hours [6]. The bioreactor was operated in a fill-draw mode. The SBR configuration comprises of five treatment phases starting from influent filling, aeration, settling, effluent discharge to idle. Fig. 3 shows the cycle of SBR process in this study. 


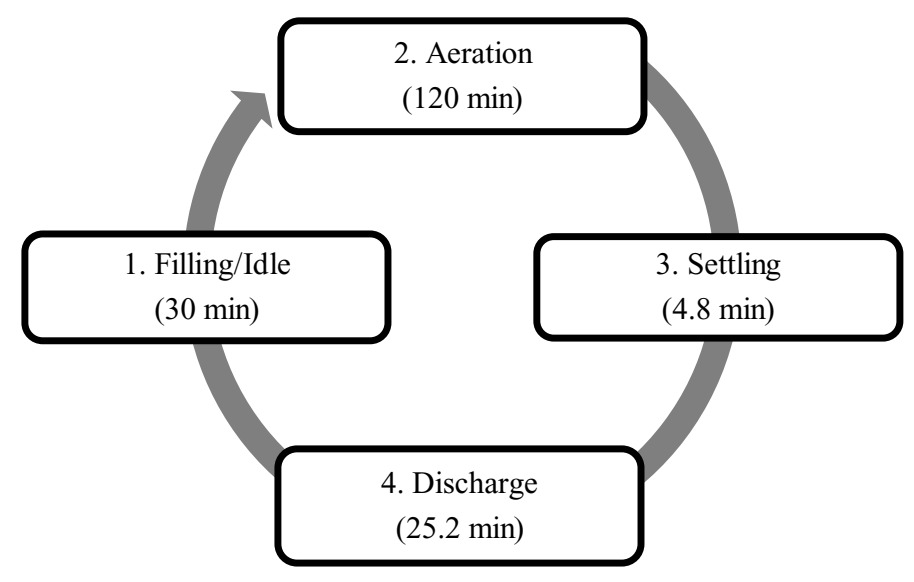

Fig. 3. SBR Operations.

The influent was introduced in the reactor through a valve positioned at the bottom of the SBR. The effluent was discharged through a discharged point at the middle of reactor height with $50 \%$ of height ratio. Air was supplied at the bottom of the reactor by a fine air bubble diffuser to give dispersed and optimum distribution of aeration. The SBR was operated at room temperature.

\subsection{Seed sludge}

Activated sludge was collected from a local wastewater treatment plant. About $900 \mathrm{~mL}$ of activated sludge was introduced initially into the bioreactor to start the process. Later, it was maintained at 50\% volume exchange ratio. The chemical oxygen demand (COD), sludge volume index (SVI), concentration of mixed liquor suspended solid (MLSS) and mixed liquor volatile suspended solid (MLVSS) were determined using APHA Standard Method [5].

\subsection{Synthetic wastewater}

The reactors were fed with the acetate-containing synthetic wastewater. The composition of synthetic wastewater was based on [6] which comprise of medium $\mathrm{X}$ and medium $\mathrm{Y}$. Medium $\mathrm{X}$ consists of $\mathrm{NaAc}, \mathrm{MgSO}_{4} .7 \mathrm{H}_{2} \mathrm{O}$ and $\mathrm{KCl}$. Medium $\mathrm{Y}$ consists of $\mathrm{NH}_{4} \mathrm{Cl}$, $\mathrm{K}_{2} \mathrm{HPO}_{4}, \mathrm{KH}_{2} \mathrm{PO}_{4}$. The trace element solution was also dosed.

\subsection{Settling velocity}

Settling velocity of the AGS and AGSM were determined using gravitational method. AGS and AGSM with different diameters ranging between $0.2-0.6 \mathrm{~mm}$ were prepared. An individual granule was selected and delicately placed in a cylinder filled with water to a height of $40 \mathrm{~cm}$, and then the settling velocity of individual granule was estimated base on teh time taken to reach the bottom. The materials and equipments needed are; (i) a measuring cylinder with $6.5 \mathrm{~cm}$ diameter and $40 \mathrm{~cm}$ depth fill with distilled water, (ii) a stopwatch (to record the settling time of granules), (iii) AGS, (iv) AGSM and (v) tweezers. Fig. 4 illustrates the experimental set-up to determine the settling velocity of AGS and AGSM in this study. 

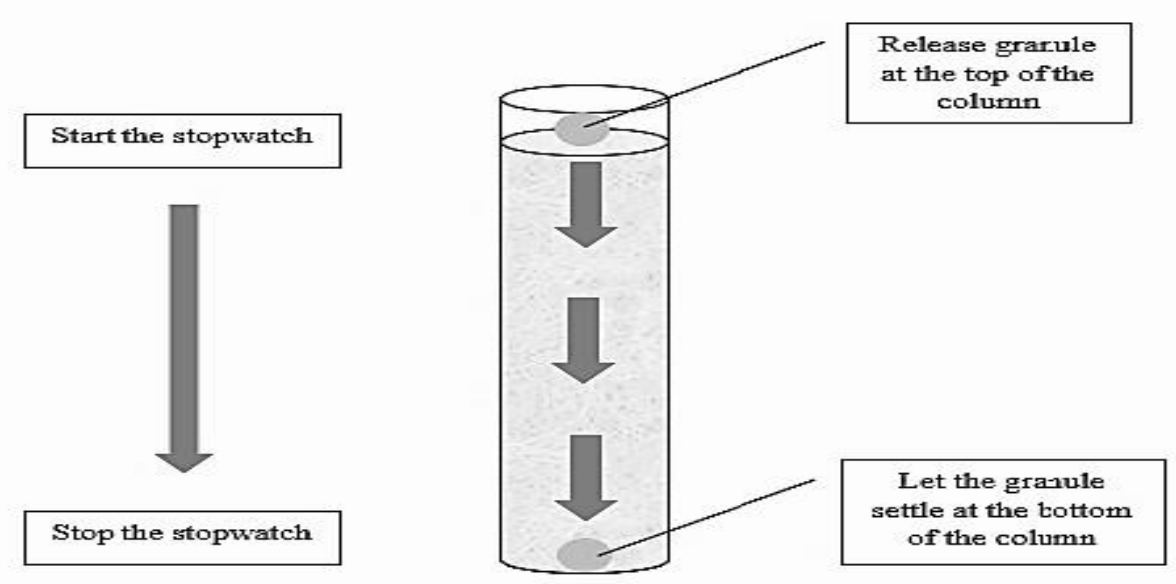

Fig. 4. Settling Velocity Procedure.

\subsection{Elemental composition determination}

The elemental composition of AGS and AGSM were determined using X-ray Fluorescence (XRF). Prior analysis, samples were dried at $110{ }^{\circ} \mathrm{C}$ for $6 \mathrm{hr}$ in an oven and then followed by drying in a muffle furnace at $600{ }^{\circ} \mathrm{C}$ for $60 \mathrm{~min}$. Samples $(\sim 2 \mathrm{~g})$ was dried in a muffle furnace at $600{ }^{\circ} \mathrm{C}$ for $2 \mathrm{hr}$ to determine the ratio of inorganic and organic matters.

\section{Results and Discussions}

\subsection{Settling velocity of AGS and AGSM}

Fig. 5 shows the settling velocity profile of AGS and AGSM for size $>0.2<0.4 \mathrm{~mm}$ of each granules. During early stage (day-14 to day-19), the result shows that the AGS took a shorter time to settle at the bottom compared to AGSM. The ration of MLVSS to MLSS of inoculum was about $60 \%$. This probably due to the adjustment and acclimatization of AGSM to the new enrichment medium and hence has not contributed to the the biomass weight and density.

On day-20, AGSM starts to increase their settling properties by having settling velocity of $23.1 \mathrm{~m} / \mathrm{h}$ with MLSS of $12.6 \mathrm{mg} / \mathrm{L}$ and continued increasing up to day-30 with settling velocity of $30.5 \mathrm{~m} / \mathrm{h}$ and MLSS of $15.7 \mathrm{mg} / \mathrm{L}$. The increment is due to the accumulation of molasses into AGS and increase of biomass and density of AGSM after succesfully adapted to the new environment.

AGS showed good adaptability to molasses and and AGSM performed good settleability which favours solid-liquid separation in the SBR. [1-2] reported good settling behaviour and biomass retention of various aerobic granules. These maybe due to the inhabitation of different biomass with different conditions of bioreactor especially in nutrients and carbon sources consumptions. 


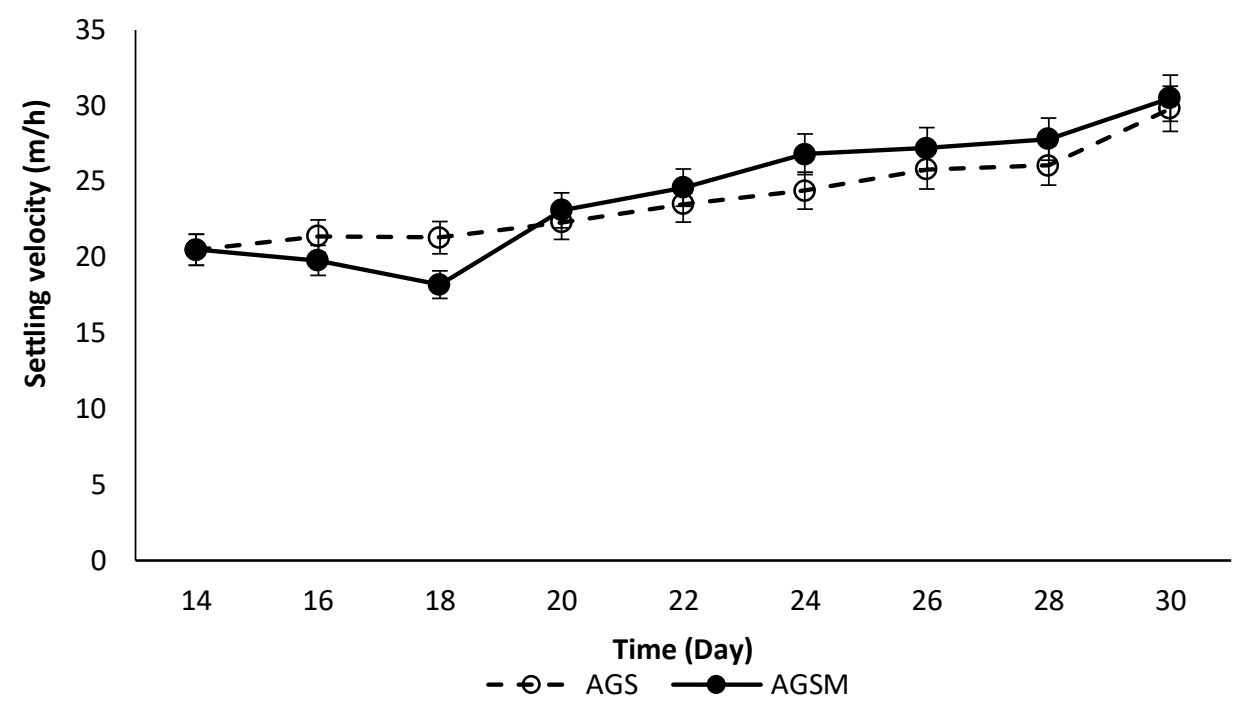

Fig. 5. Settling Velocity of AGS and AGSM.

\subsection{Elemental compositions of AGS and AGSM}

Fig. 6 shows the XRF results of AGS and AGSM in this study. The XRF results shows that main chemical composition of AGS is palladium (Pd) with $74 \%$, followed by hafnium (Hf) with 13\%, calcium (Ca) $4.7 \%$ and small amount of other elements like zinc ( $\mathrm{Zn}$ ), ferum $(\mathrm{Fe})$, potassium $(\mathrm{K})$ and rhenium $(\mathrm{Re})$. The various chemicals were detected in AGS due to the samples which are collected from a biological tank from a wastewater treatment plant in a latex processing industry.

According to [7], there were various chemicals and materials used in industries such as in latex processing. Palladium and hafnium were used in numerous application including textiles manufacturing [8]. The XRF results indicate that the main chemical composition of AGSM is Hf with 74\%. Other chemical compositions detected in AGSM are K with $16.4 \%$, Re with $13 \%$, Ca with $3.6 \%$ and small amount of other elements including $\mathrm{Fe}, \mathrm{Pd}$, Os and $\mathrm{Zn}$. In another study, it was found that about seven types of elements present in molasses wastewater including $\mathrm{Fe}, \mathrm{Zn}$, manganese $(\mathrm{Mn})$, copper $(\mathrm{Cu})$, chromium $(\mathrm{Cr})$, cadmium $(\mathrm{Cd})$ and cobalt $(\mathrm{Co})$. Each compounds were detected at a range from $0.08 \mathrm{mg} / \mathrm{L}$ to $348 \mathrm{mg} / \mathrm{L}$ in molasses wastewater samples [9].

Overall, seven chemical elements were detected in AGS, while eight composition elements are found in AGSM. Similar elements were detected in both the AGS and AGSM. Different elemental ratio shows that the acclimatization and enhancement of molasses wastewater on the cultivated AGS has influenced the chemical composition of granules formed depend on the nutrients available in the molasses wastewater. This study gives an insight on the potential of using AGS to treat molasses wastewater. Molasses wastewater contains high organic and inorganic compounds makes it difficult to achieve high solid:liquid separation and colour removal. With the the abundant nutrients present in molasses wastewater, the agglomeration and growth of microorganisms in AGS can be enriched. Hence, employing AGS in molasses wastewater could favour biomass accumulation and colour removal problem in molasses processing industry. 


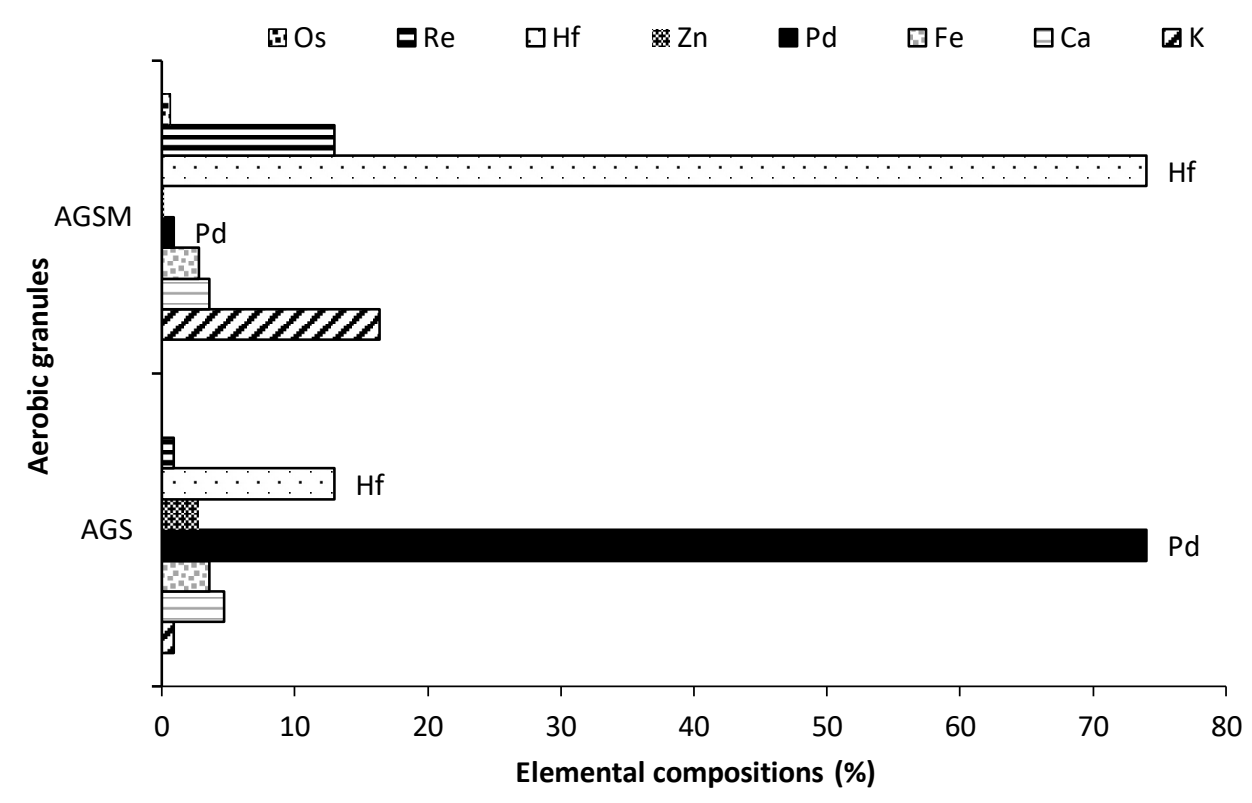

Fig. 6. Chemical Compositions in AGS and AGSM.

\section{Conclusions}

This study demonstrated that aerobic granular sludge could be cultivated in nutrient rich molasses wastewater. After 30-day operation, stable granules with greater settling properties were obtained with average size $\sim 0.4 \mathrm{~mm}$. With enhanced granulation in molasses wastewater, the settling performance of the granules formed gradually increased, as indication by a decreased sludge volume index and an increased settling velocity. The formed granules was regular, spherical and appear in black colour. The dark colour granules indicates the accumulation and enrichment of molasses in the granules. XRF analysis gives detailed various elemental compositions in the cultivated and enhanced granules. This suggests that the aerobic granular sludge served as a good starting inoculum for the colour removal treatment of molasses wastewater. Further studies will have to be resolved in a future investigation.

Authors wish to thank the Ministry of Higher Education Malaysia for the Fundamental Research Grant Scheme (FRGS) No. 9003-00386, RACE Grant (9017-0009), and Universiti Malaysia Perlis for the financial aid through Postgraduate Academic Activities Fund (PAAF).

\section{References}

1. A. Nor-Anuar., Z. Ujang, M.C.M. Van Loosdrecht, M.K. De Kreuk. Wat. Sci. and Tech. 56(7), 55-63 (2007)

2. F.A. Dahalan, N. Abdullah, A. Yuzir, G. Olsson, M. Hamdzah, M.F.M. Din, S.A. Ahmad, K.A. Khalil, A.N. Anuar, Z.Z. Noor, Z. Ujang. Bio. Tech. 181, 291-296 (2015)

3. P. P. Kalbar, S. Karmakar, S.R. Asolekar. Wat. Env. J. 27(2), 261-268 (2013) 
4. S.S. Adav, D.J. Lee, K.Y. Show, J.H. Tay. Bio. Adv. 26(5), 411-423 (2008)

5. APHA (American Public Health Association). Standard methods for the examination of water and wastewater. (American Public Health Association, Washington DC, 2005)

6. A. Nor-Anuar. Development of aerobic granular sludge technology for domestic wastewater treatment in hot climate. Unpublished Ph.D dissertation. (Universiti Teknologi Malaysia, 2008)

7. E., Morgenroth, T. Sherden, M.C.M. Van Loosdrecht, J.J. Heijnen, P.A. Wilderer. Wat. Res. 31(12), 3191-3194 (1997)

8. F.A. Dahalan, N. Abdullah, A. Yuzir, G. Olsson, M. Hamzah, M.F.M. Din, Ujang, Z. Bio. Tech. 181, 291-296 (2015)

6. T. Akabane. Int. Poly. Sci. and Tech. 43(5), T45 (2016)

7. U. Marga, S. Yanti, S. Made, P. Tita, I. Dian, K. Makuuchi, F. Yoshii. Production of surgical gloves from low extractable protein. RVNRL No. JAERI-CONF. 2001-005 (2001)

8. American Elements. Hafnium Nitrate Solution. Retrieved from https://www.americanelements.com/hafnium nitrate-solution-15509-05-4 (2018)

9. I.S. Thakur. Industrial biotechnology: problems and remedies. (IK International Pvt Ltd., New Delhi, 2010) 